there enough spare land for food production in developing countries, and will the limits of Earth's resources control human numbers?

The journal's stated aims are exceptionally broad, but are intended to provide a multidisciplinary appraisal of the theory and practice of sustainable development. It achieves this with an interesting range of generally high-quality articles. Its international remit is realized, although with an emphasis on developing countries. Topics addressed to date include environmental and socio-economic aspects of forest land allocation and deforestation in Vietnam, the ecological impacts of Chile's neo-liberal policies and tropical coastal organisms as indicators of mercury pollution in India.

Although such research could be published in a range of outlets, the thread of sustainable development is woven through the papers and the journal stands up well as an integrated whole. This is reinforced by the inclusion of work related to policy (renewed efforts by the Organisation for Economic Co-operation and Development to address sustainable development are documented) and theory. Specific methodologies offering the potential to chart progress towards (or away from) sustainability are addressed, with a robust defence of ecological footprinting - a technique to try to link the land-area resource requirements to per capita consumption for different societies. Training initiatives also fall within the journal's remit, with an account of a simulation model for vulnerability to climate change.

Each issue comprises between four and six papers of variable length (submission to acceptance times are typically six to ten months), followed by a book review. Given the wide range of subjects encompassed, any particular issue of the journal can touch on only a few, but the selections presented are well balanced and will appeal to those wishing to keep abreast of current sustainability-related topics. Special issues look set to become a feature, with the first - "Local knowledge in the tropics: Relevance to conservation and management" - being the proceedings of the annual meeting of the Association for Tropical Biology.

Environment, Development and Sustainability was launched in the last year of a century during which, by any definition, development had become increasingly unsustainable. Whether it will document continued decline or positive progress remains to be seen, but the journal shows the potential to offer a valuable commentary.

David R. Brown is at the School of Environmental

Sciences, University of East Anglia, Norwich

NR4 7TJ, UK.

www.wkap.nl/journalhome.htm/1387-585X

\section{Room for one more on board?}

\section{Advanced Synthesis \& Catalysis \\ executive editor Joe P. Richmond \\ Wiley-VCH. 8/yr. 428 euros, $\$ 498$ \\ (institutional) \\ Amir Hoveyda}

Catalysis and synthesis are two of the most important areas of investigation in modern organic chem-

istry. These fields have critical implications for medical and pharmaceutical research as well as for materials science. Not surprisingly, many outstanding, internationally recognized journals dedicate a notable portion of their pages to describing advances in these areas. Journal of the American Chemical Society and Angewandte Chemie are among at least ten respected publications that regularly cover the latest research in catalysis and synthesis. Such publications deal with novelty of approach and with issues of practicality, scale and environmental friendliness.

It has become an accepted dictum that a new method in synthesis must strive to satisfy some or all of the above criteria; and papers introducing a new catalyst or synthesis method generally include discussions of these issues. After all, some of the most important aspects of catalysis concern issues of economy and the environment. Papers in the journals mentioned above frequently cover attempts to develop recyclable supported catalysts, those that do not require a solvent, or processes that have a high turnover number and/or frequency. And several journals regularly publish symposia-in-print that cover exciting new related topics.

It is therefore difficult to understand immediately what niche Advanced Catalysis \& Synthesis is expected to fill. The publishers state that the new publication focuses on "chemical reactions that are economical, safe, environmentally benign, resource- and energy-saving". But such attributes have always been part of the yardstick by which papers in catalysis and synthesis are measured. For example, the incisive and thought-provoking review article on catalytic kinetic resolution that appears in the first issue could easily have been accommodated in Angewandte Chemie, a journal produced by the same publisher - one might argue that the latter venue would have allowed the article to reach a wider audience.

Most of the remaining reviews and communications in the journal's first four issues address questions of efficiency and selectivity. A few others are directed more towards the practical aspects of synthesis and catalysis, but all could have been submitted to any of several existing journals.

Perhaps the most striking feature of this new publication is its illustrious editorin-chief and editoral board; a more respected and better-qualified group would have been difficult to assemble. Such a talented and accomplished editorial ensemble clearly have a valid reason for supporting the journal. But if there is such a mission, it has unfortunately not yet emerged from the first 400 pages of Advanced Catalysis \& Synthesis. Furthermore, in thinking about the need for new journals in these important and well-represented subdisciplines of chemistry, and considering the truncated budgets and rising costs that face most of our libraries, one is reminded of the words of the great twentieth-century architect, Ludwig Mies van der Rohe, that "less is more".

Amir H. Hoveyda is in the Department of Chemistry, Merkert Chemistry Center, Boston College, Chestnut Hill, Massachusetts 02467, USA.

\section{Forum for the genomic onslaught}

\section{GenomeBiology.com}

editor Theodora Bloom

BioMed Central. \$300, 190 (library, print only), \$130, £80 (laboratory, print \& online), \$120, £75 (individual, print \& online); \$95,

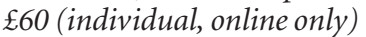

\section{John D. McPherson}

The past few years have witnessed an explosion of publicly accessible data on the genomes of a wide variety of organisms. These data sets now include physical maps and DNA sequence and annotation of complete genomes. The analyses of such data sets are increasingly large and complex. To better serve the research community, many journals offer a complementary website providing supplemental material that is inappropriate for a printed format. 
These supplemental data include additional details on large-scale analysis methods and, often, access to the data themselves. GenomeBiology.com is one of the new breed of journals that publish primarily in a webbased format with a supplemental printed journal profiling selected articles and summarizing or merely indexing others with reference to the website.

The main objective of GenomeBiology. com is to provide a forum for articles concerning biology that utilize the wealth of genomic data now available, or in their words, "Biology for the post-genomic era". The website/journal aims to be a guide to these genomic resources and to present the impact of the data on biological research in the form of timely reviews and research articles. An extensive international advisory board with expertise in a wide range of organisms and disciplines is charged with determining the appropriateness of submitted articles for the scope of the journal, which is meant to cover all aspects of genomic research, including molecular and cellular biology, genomics, proteomics, bioinformatics, computational biology, large-scale sequence analysis, comparative biology and molecular evolution. The journal succeeds very well in providing a rich resource for publication in these data-intensive research areas, which many traditional journals cannot readily support.

GenomeBiology.com aims for speed of publication, encouraging the use of a direct, web-based upload submission system and a promise of quick review. Although there are no length limits for the web-based articles, most remain succinct and understandable. The primary use of the website as

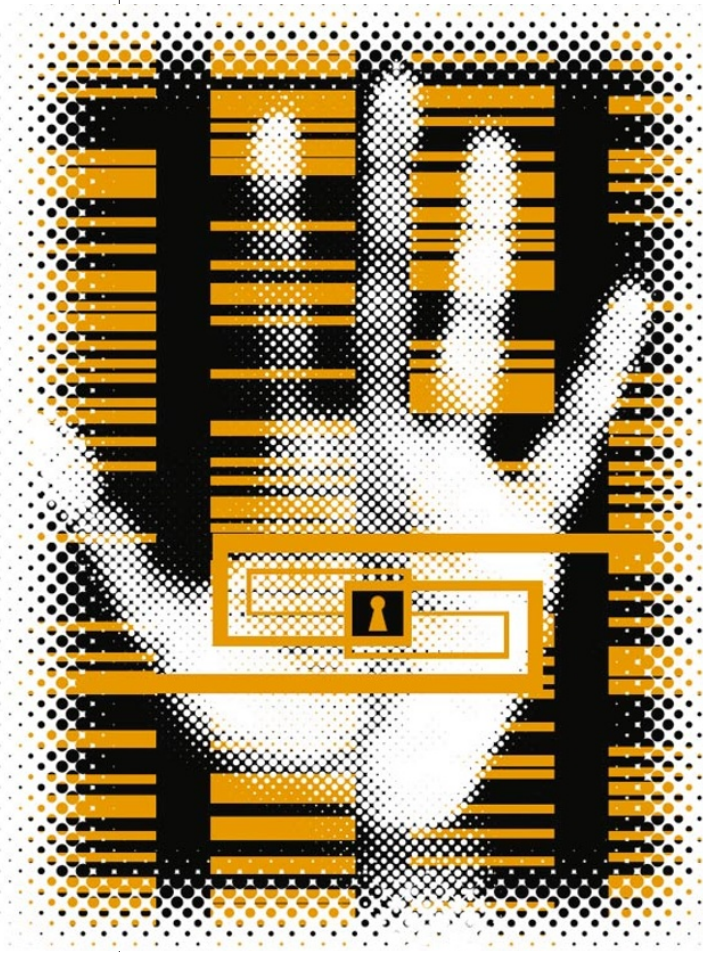

a publication forum allows the ready inclusion of greater numbers of more complex images and also supports movies and access to raw data.

An additional component of the website is a depository for non-refereed articles with no access restrictions for the research community. Authors are solely responsible for the content of these articles; however, the articles are screened to ensure their relevance to the scope of the journal and to prevent abuse of this service.

Although it is premature to declare this the post-genomic era, GenomeBiology.com recognizes the need for journals to adapt to today's data-intensive biological research. Web support for journals, and indeed webbased journals, are essential for providing comprehensive presentation of research material. GenomeBiology.com offers research articles of broad interest and insightful reviews. It is an excellent guide to the overwhelming onslaught of genomic and proteomic data and will flourish if its current trend continues.

John D. McPherson is in the Department of Genetics, Washington University School of Medicine, St Louis, Missouri 63108, USA. http://genomebiology.com

\section{On the high side of life}

High Altitude Medicine \& Biology

editor-in-chief John B. West

Mary Ann Liebert. 4/yr. \$300 (institutional), $\$ 191$ (individual)

\section{Ronald J. White and Jancy C. McPhee}

Over the past 20 years, interest in the highaltitude life sciences has grown steadily and become increasingly diverse and interdisciplinary. An idea of this can be gained by considering the range of topics — from interleukin-6 levels in high-altitude pulmonary oedema to evolutionary physiology - that are discussed at the International Hypoxia Symposia and other focused scientific meetings.

This growing interest has led to the creation of High Altitude Medicine \& Biology, a journal with an intentionally broad scope that serves the varied needs of this specialized medical and scientific community. Like the field, the editorial board is diverse, both in expertise and nationality, with world-class members from 17 countries. This speciality journal is off to a good start.

The journal's scope includes highaltitude diseases, physiology, pathology, comparative biology, anthropology, evolutionary biology, and human and animal ecology. This breadth is reflected in the articles in the first few issues, which range

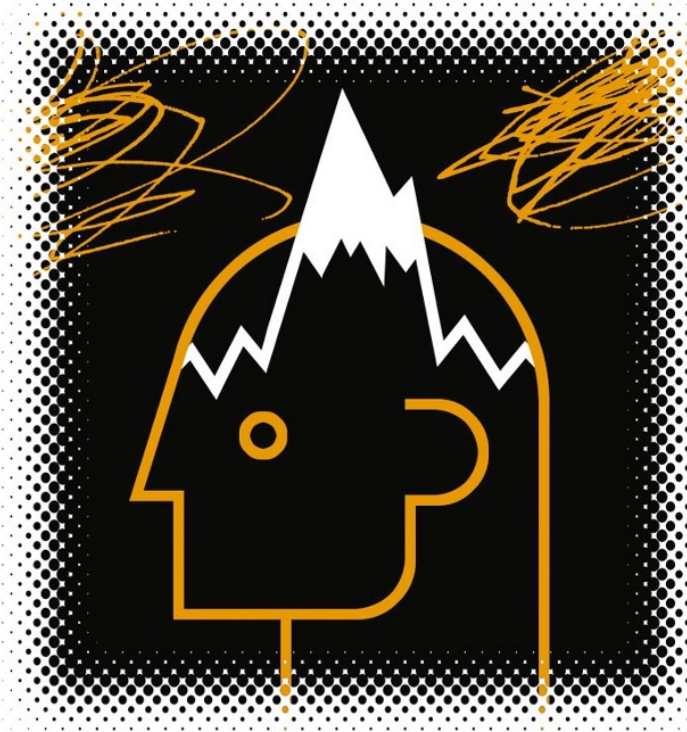

from fundamental gene-based studies to ethnobotany. In addition, the journal hopes to deal with such interesting issues as the practical problems in medicine that develop as more and more people commute to work from low elevations to high altitude.

High Altitude Medicine \& Biology does not aim to compete with well-established discipline-based journals, but rather to provide a home for multidisciplinary work, or for articles that do not fit within the scope of existing journals. This policy is wise but presents its own challenge - that of drawing the highest-quality manuscripts in this eclectic field. The editors hope that the journal will actually spur research in the diverse areas within its scope. Only time will tell whether they succeed in this task while maintaining the current quality.

This is an attractive journal, with highquality print, clear figures, good photographs and a nice layout. Published manuscripts are reviewed and accepted in a reasonable time - about two months - and publication currently follows acceptance rapidly. The articles average about eight pages in length.

A special feature is a section entitled "Sightings", which gives readers interesting glimpses of articles from journals that may be far from their normal reading list. Another feature, "High Altitude Web", provides information on where to look on the Internet for useful information. The journal has space for letters to the editor and book reviews. Interesting case studies, reviews, historical articles and intermittent poetry are all included. Occasionally, abstracts from symposia (such as the International Hypoxia Symposia) are planned.

The journal has already met with some success. It is being indexed by Index Medicus and citations are available through Medline. Additionally, it has been selected as the official journal of the International Society 